\title{
Kalman Filtering for Discrete Stochastic Systems with Multiplicative Noises and Random Two-Step Sensor Delays
}

\author{
Dongyan Chen, ${ }^{1}$ Yonglong $\mathrm{Yu},{ }^{1}$ Long $\mathrm{Xu},{ }^{1}$ and Xiaohui Liu ${ }^{2,3}$ \\ ${ }^{1}$ Department of Applied Mathematics, Harbin University of Science and Technology, Harbin 150080, China \\ ${ }^{2}$ Department of Computer Science, Brunel University London, Uxbridge, Middlesex UB8 3PH, UK \\ ${ }^{3}$ Faculty of Engineering, King Abdulaziz University, Jeddah 21589, Saudi Arabia
}

Correspondence should be addressed to Dongyan Chen; dychen_2004@hotmail.com

Received 4 January 2015; Accepted 25 January 2015

Academic Editor: Zidong Wang

Copyright (C) 2015 Dongyan Chen et al. This is an open access article distributed under the Creative Commons Attribution License, which permits unrestricted use, distribution, and reproduction in any medium, provided the original work is properly cited.

\begin{abstract}
This paper is concerned with the optimal Kalman filtering problem for a class of discrete stochastic systems with multiplicative noises and random two-step sensor delays. Three Bernoulli distributed random variables with known conditional probabilities are introduced to characterize the phenomena of the random two-step sensor delays which may happen during the data transmission. By using the state augmentation approach and innovation analysis technique, an optimal Kalman filter is constructed for the augmented system in the sense of the minimum mean square error (MMSE). Subsequently, the optimal Kalman filtering is derived for corresponding augmented system in initial instants. Finally, a simulation example is provided to demonstrate the feasibility and effectiveness of the proposed filtering method.
\end{abstract}

\section{Introduction}

The filtering problem has been a mainstream research topic in the control theory due to its wide and important engineering applications such as signal processing, econometrics communication, guidance, navigation, and control of vehicles [14]. Kalman filtering, also known as linear optimal quadratic estimation, has attracted much research interests due to its good filtering performance and simple filtering structure $[5,6]$. In [7], based on the minimum mean square error (MMSE) principle and the projection theory, the traditional Kalman filtering algorithm has been proposed for a class of linear discrete stochastic systems. Subsequently, the Kalman filtering problems have been widely investigated for different systems $[8,9]$. For the nonlinear model, the theoretical results of the extended Kalman filter (EKF) have been proposed and applied in many practical engineering problems [1013]. For example, in [14], the EKF algorithm has been employed to deal with the mobile robot localization problem with intermittent measurements, where the cases of missing measurements and uncertainties have been addressed. For the microelectromechanical systems, a new terminal slidingmode control scheme has been designed in [15] by using the EKF observer.

During the processes of signal measurement, transmission, and computation, the sensor delays are frequently encountered and are inevitable especially in the networked systems [16-21]. The existence of the sensor delays would deteriorate the filtering accuracy and even influence the control system performance [22-26]. Hence, it is not a surprise that a great number of results have been reported to handle the Kalman filtering problems with the sensor delays [8, 9, 27]. To mention a few, the optimal Kalman filtering problem has been investigated in [8] for linear discrete system with sensor delays, packet dropouts, and uncertain observations. It has been shown that a unified augmentation method has been proposed in [8] by applying the projection theory and recursive projection formula, which can reduce the amount of correlated parameters. Motivated by the method in [8], the optimal Kalman filtering algorithm has been given in [9] for the systems with random sensor delays. Based on the unbiasedness and MMSE of the optimal Kalman filtering, 
the recursive optimal Kalman filtering approaches have been developed in $[27,28]$ for linear stochastic systems with random sensor delays. Compared with the methods in [27, 28], the developed approach in [9] can reduce the amount of correlated parameters when tackling the optimal filtering problem for systems with random sensor delays.

Note that a great deal of effort has been devoted to address the problems of optimal Kalman filtering with one-step sensor delay in the past years $[29,30]$. Nevertheless, it should be pointed out that randomly occurring two-step sensor delays are also encountered in some networked systems [31]. Recently, the case of the noisy observation measurements with random one-step or two-step sample delays has been investigated and a novel unscented filtering algorithm has been given in [31] for a class of nonlinear discrete-time stochastic systems. On the other hand, it is necessary to deal with the multiplicative noises when designing the Kalman filtering [32-34]. The optimal nonfragile Kalman-type filtering problem has been investigated in [32] for a class of systems with multiplicative noises, finite-step autocorrelated measurement noises, and multiple packet dropouts, where the state-dependent multiplicative noises have been used to account for the stochastic uncertainties. In [33], a new nonlinear filter has been constructed to attenuate the effects from the multiplicative noises and the signal quantization. In [34], the linear minimum mean square estimator has been designed for linear discrete-time systems with state and measurement multiplicative noises and Markov jumps on the parameters. It is worth pointing out that, however, the optimal Kalman filtering problem has not been investigated for linear stochastic systems with multiplicative noises and random two-step sensor delays yet.

Motivated by the above discussions, in this paper, we aim to discuss the problem of optimal Kalman filtering for linear discrete stochastic system with multiplicative noises and random two-step sensor delays. The state-dependent multiplicative noises are considered to account for the stochastic uncertainties. The phenomena of two-step sensor delays may happen in data transmission and are described by using three Bernoulli distributed random variables with known conditional probabilities. Based on the MMSE estimation principle, the optimal Kalman filtering problem has been discussed for system with multiplicative noises and random two-step sensor delays. Firstly, we consider a general case for the original system where $k \geq 3$. By using the state augmentation approach and the projection theory, the optimal Kalman filtering algorithm has been given for augmented system. Then, the optimal Kalman filtering for the original system can be obtained easily. Secondly, we discuss the initial case when $k=1(k=2)$ and give some parameters to help algorithm developments. The main contributions of this paper can be highlighted as follows: (1) the system model is more general where the multiplicative noises and randomly occurring two-step sensor delays are considered simultaneously and (2) a new Kalman filter is designed to handle the addressed complex phenomena. Finally, an illustrative example is provided to verify the feasibility and effectiveness of the proposed result.
The rest of this paper is organized as follows. In Section 2, the problem addressed is formulated and some preliminaries are briefly introduced. In Section 3, a new Kalman filtering algorithm is proposed to deal with the systems with multiplicative noises and random two-step sensor delays and the explicit form of the filter gain is given. In Section 4, an illustrative example is used to show the effectiveness of the proposed filtering method. Finally, we provide the conclusions in Section 5.

Notations. The notations used throughout the paper are standard. $\mathscr{R}^{n}$ and $\mathscr{R}^{n \times m}$ denote the $n$-dimensional Euclidean space and the set of all $n \times m$ matrices, respectively. For a matrix $P$, the $P^{T}$ and $P^{-1}$ represent its transpose and inverse, respectively. $\mathbb{E}\{x\}$ stands for the expectation of a stochastic variable $x$. $\operatorname{diag}\left\{P_{1}, P_{2}, \ldots, P_{n}\right\}$ stands for a block-diagonal matrix with matrices $P_{1}, P_{2} \ldots, P_{n}$ on the diagonal. $I$ and 0 represent the identity matrix and the zero matrix with appropriate dimensions, respectively. Matrices are assumed to be compatible with algebraic operations if their dimensions are not explicitly stated.

\section{Problem Formulation and Preliminaries}

In this paper, we consider the following class of discrete uncertain stochastic systems with multiplicative noises and random two-step sensor delays:

$$
\begin{gathered}
x_{k+1}=\left(A_{k}+A_{s, k} \xi_{k}\right) x_{k}+B_{k} \omega_{k} \\
z_{k}=\left(C_{k}+C_{s, k} \eta_{k}\right) x_{k}+v_{k} \\
y_{k}=\sum_{i=0}^{\min \{k-1,2\}} \gamma_{k}^{i} z_{k-i},
\end{gathered}
$$

where $x_{k} \in \mathscr{R}^{n}$ is the system state vector to be estimated, $z_{k} \in \mathscr{R}^{m}$ is measured output, and $y_{k} \in \mathscr{R}^{m}$ is measurement received by the sensor. $\omega_{k} \in \mathscr{R}^{n}$ and $\nu_{k} \in \mathscr{R}^{m}$ are uncorrelated white noises with zero means and variance matrices $Q_{\omega_{k}} \geq 0$ and $Q_{\gamma_{k}}>0 . \xi_{k}$ and $\eta_{k}$ are multiplicative noises with zero means and unity covariances and are uncorrelated with other noise signals. $A_{k}, A_{s, k}, B_{k}, C_{k}$, and $C_{s, k}$ are known real time-varying matrices with appropriate dimensions.

The random variables $\gamma_{k}^{i}$ obey the Bernoulli distribution and have the following statistical properties:

$$
\begin{gathered}
\operatorname{Prob}\left\{\gamma_{k}^{i}=1\right\}=\mathbb{E}\left\{\gamma_{k}^{i}\right\}=\alpha_{i}, \\
\operatorname{Prob}\left\{\gamma_{k}^{i}=0\right\}=1-\mathbb{E}\left\{\gamma_{k}^{i}\right\}=1-\alpha_{i},
\end{gathered}
$$

where $\alpha_{i} \in[0,1](i=0,1,2)$ are known positive scalars. Assume that $\gamma_{k}^{i}$ are mutually independent of other noise signals.

Remark 1. As in [31], for $k \geq 3$, if $\gamma_{k}^{0}=1, \gamma_{k}^{1}=0$, and $\gamma_{k}^{2}=0$ in model (3), one has $y_{k}=z_{k}$; that is, the sensor receives the data at the time instant $k$; if $\gamma_{k}^{0}=0, \gamma_{k}^{1}=1$, and $\gamma_{k}^{2}=0$, one has $y_{k}=z_{k-1}$; that is, there exists the one-step time delay; if $\gamma_{k}^{0}=0, \gamma_{k}^{1}=0$, and $\gamma_{k}^{2}=1$, one has $y_{k}=z_{k-2}$; that is, there 
exists the two-step time delays. For special cases, when $k=1$, the sensor receives the signal on time, $y_{1}=\gamma_{1}^{0} z_{1}$ with $\gamma_{1}^{0}=1$. When $k=2$, the sensor receives the signal on time or the onestep sensor delay occurs, $y_{2}=\gamma_{2}^{0} z_{2}+\gamma_{2}^{1} z_{1}$; here $\gamma_{2}^{0}=1, \gamma_{2}^{1}=0$ or $\gamma_{2}^{0}=0$, and $\gamma_{2}^{1}=1$; that is, $\gamma_{2}^{0}+\gamma_{2}^{1}=1$. In other words, these Bernoulli distributed variables satisfy $\sum_{i=0}^{\min \{k-1,2\}} \gamma_{k}^{i}=1$ for all $k \geq 1$.

Assumption 2. The initial state $x_{0}$ is uncorrelated with other noise signals, and

$$
\mathbb{E}\left\{x_{0}\right\}=\mu_{0}, \quad \mathbb{E}\left\{\left(x_{0}-\mu_{0}\right)\left(x_{0}-\mu_{0}\right)^{T}\right\}=P_{0} .
$$

Without loss of generality, for $k \geq 3$, we can rewrite (3) as follows:

$$
y_{k}=\gamma_{k}^{0} z_{k}+\gamma_{k}^{1} z_{k-1}+\gamma_{k}^{2} z_{k-2} .
$$

By defining $\vec{x}_{k}=\left[\begin{array}{lll}x_{k}^{T} & x_{k-1}^{T} & x_{k-2}^{T}\end{array}\right]^{T}$, the systems (1), (2), and (6) can be rewritten as the following compact form:

$$
\begin{gathered}
\vec{x}_{k+1}=\vec{\Phi}_{k} \vec{x}_{k}+\vec{B}_{k} \vec{\omega}_{k} \\
\vec{y}_{k}=\vec{H}_{k} \vec{x}_{k}+\vec{\Lambda}_{k} \vec{v}_{k},
\end{gathered}
$$

where

$$
\begin{aligned}
& \vec{y}_{k}=y_{k}, \quad \vec{\omega}_{k}=\omega_{k}, \quad \vec{v}_{k}=\left[\begin{array}{c}
v_{k} \\
v_{k-1} \\
v_{k-2}
\end{array}\right] \text {, } \\
& \vec{\Phi}_{k}=\vec{A}_{k}+\xi_{k} \vec{A}_{s, k}, \quad \vec{A}_{k}=\left[\begin{array}{ccc}
A_{k} & 0 & 0 \\
I & 0 & 0 \\
0 & I & 0
\end{array}\right], \\
& \vec{A}_{s, k}=\left[\begin{array}{ccc}
A_{s, k} & 0 & 0 \\
0 & 0 & 0 \\
0 & 0 & 0
\end{array}\right], \quad \vec{B}_{k}=\left[\begin{array}{c}
B_{k} \\
0 \\
0
\end{array}\right] \text {, } \\
& \vec{H}_{k}=\vec{\Lambda}_{k} \vec{C}_{k}+\vec{\Lambda}_{k} \vec{C}_{s, k} \vec{\eta}_{k}, \quad \vec{\Lambda}_{k}=\left[\begin{array}{c}
\gamma_{k}^{0} I \\
\gamma_{k}^{1} I \\
\gamma_{k}^{2} I
\end{array}\right]^{T}, \\
& \vec{C}_{k}=\left[\begin{array}{ccc}
C_{k} & 0 & 0 \\
0 & C_{k-1} & 0 \\
0 & 0 & C_{k-2}
\end{array}\right], \quad \vec{C}_{s, k}=\left[\begin{array}{ccc}
C_{s, k} & 0 & 0 \\
0 & C_{s, k-1} & 0 \\
0 & 0 & C_{s, k-2}
\end{array}\right], \\
& \vec{\eta}_{k}=\left[\begin{array}{ccc}
\eta_{k} I & 0 & 0 \\
0 & \eta_{k-1} I & 0 \\
0 & 0 & \eta_{k-2} I
\end{array}\right] \text {. }
\end{aligned}
$$

For convenience of the subsequent developments, set

$$
\begin{gathered}
\bar{\Phi}_{k}=\mathbb{E}\left\{\vec{\Phi}_{k}\right\}=\vec{A}_{k}, \quad \Delta \Phi_{k}=\vec{\Phi}_{k}-\bar{\Phi}_{k}=\xi_{k} \vec{A}_{s, k}, \\
\vec{\Lambda}_{k}=\gamma_{k}^{0} \Lambda_{0}+\gamma_{k}^{1} \Lambda_{1}+\gamma_{k}^{2} \Lambda_{2}, \\
\bar{\Lambda}_{k}=\mathbb{E}\left\{\vec{\Lambda}_{k}\right\}=\left[\begin{array}{lll}
\alpha_{0} I & \alpha_{1} I & \alpha_{2} I
\end{array}\right], \\
\Lambda_{0}=\left[\begin{array}{lll}
I & 0 & 0
\end{array}\right], \quad \Lambda_{1}=\left[\begin{array}{lll}
0 & I & 0
\end{array}\right], \\
\Lambda_{2}=\left[\begin{array}{lll}
0 & 0 & I
\end{array}\right],
\end{gathered}
$$

$$
\begin{gathered}
\Delta \Lambda_{k}=\vec{\Lambda}_{k}-\bar{\Lambda}_{k}=\left[\begin{array}{c}
\left(\gamma_{k}^{0}-\alpha_{0}\right) I \\
\left(\gamma_{k}^{1}-\alpha_{1}\right) I \\
\left(\gamma_{k}^{2}-\alpha_{2}\right) I
\end{array}\right]^{T} \\
=\left(\gamma_{k}^{0}-\alpha_{0}\right) \Lambda_{0}+\left(\gamma_{k}^{1}-\alpha_{1}\right) \Lambda_{1}+\left(\gamma_{k}^{2}-\alpha_{2}\right) \Lambda_{2}, \\
\bar{H}_{k}=\mathbb{E}\left\{\vec{H}_{k}\right\}=\bar{\Lambda}_{k} \vec{C}_{k}, \\
\Delta H_{k}=\vec{H}_{k}-\bar{H}_{k}=\left(\vec{\Lambda}_{k}-\bar{\Lambda}_{k}\right) \vec{C}_{k}+\vec{\Lambda}_{k} \vec{C}_{s, k} \vec{\eta}_{k}, \\
\vec{\eta}_{k}=\eta_{k} N_{0}+\eta_{k-1} N_{1}+\eta_{k-2} N_{2}, \\
N_{0}=\operatorname{diag}\{I, 0,0\}, \quad N_{1}=\operatorname{diag}\{0, I, 0\}, \\
N_{2}=\operatorname{diag}\{0,0, I\} .
\end{gathered}
$$

Then, it is easy to obtain that

$$
\mathbb{E}\left\{\Delta \Phi_{k}\right\}=0, \quad \mathbb{E}\left\{\Delta H_{k}\right\}=0, \quad \mathbb{E}\left\{\Delta \Lambda_{k}\right\}=0 .
$$

The purpose of this paper is to design the optimal Kalman filter $\hat{x}_{k \mid k}$ for the addressed discrete uncertain stochastic systems (1)-(3) based on the observation sequence $\left\{y_{1}, y_{2}, \ldots, y_{k-1}\right\}$. Noting the relationship between the original system and the augmented system, we know $\widehat{x}_{k \mid k}=$ $\left[\begin{array}{lll}I & 0 & 0\end{array}\right] \widehat{\vec{x}}_{k \mid k}$.

\section{Main Results}

In this section, by using the projection theory, the recursion of the Kalman filtering is derived and the explicit expression of the filter gain is given.

To facilitate the subsequent developments, we introduce the following definition and lemmas.

Definition 3 (see [8]). Let $\Xi_{k}=\mathbb{E}\left\{\vec{x}_{k} \vec{x}_{k}^{T}\right\}$ be the state covariance matrix. Then, one has

$$
\Delta_{\vec{T}_{k} \vec{U}_{k}}\left(\Xi_{k}\right)=\mathbb{E}\left\{\left[\vec{T}_{k}-\mathbb{E}\left\{\vec{T}_{k}\right\}\right] \vec{x}_{k} \vec{x}_{k}^{T}\left[\vec{U}_{k}-\mathbb{E}\left\{\vec{U}_{k}\right\}\right]^{T}\right\},
$$

where $\vec{T}_{k}$ and $\vec{U}_{k}$ are time-varying stochastic matrices.

Motivated by the excellent results in [8], we can obtain the following lemmas which would be helpful for the further calculation.

Lemma 4. According to the definition of the $\vec{\Phi}_{k}$ and $\vec{H}_{k}$, one has

$$
\Delta_{\vec{\Phi}_{k} \vec{\Phi}_{k}}\left(\Xi_{k}\right)=\vec{A}_{s, k} \Xi_{k} \vec{A}_{s, k}^{T}
$$

$$
\begin{aligned}
\Delta_{\vec{H}_{k} \vec{H}_{k}}\left(\Xi_{k}\right)= & \alpha_{0}\left(1-\alpha_{0}\right) \Lambda_{0} \vec{C}_{k} \Xi_{k} \vec{C}_{k}^{T} \Lambda_{0}^{T} \\
& +\alpha_{1}\left(1-\alpha_{1}\right) \Lambda_{1} \vec{C}_{k} \Xi_{k} \vec{C}_{k}^{T} \Lambda_{1}^{T} \\
& +\alpha_{2}\left(1-\alpha_{2}\right) \Lambda_{2} \vec{C}_{k} \Xi_{k} \vec{C}_{k}^{T} \Lambda_{2}^{T}-\mathscr{A}_{k}-\mathscr{A}_{k}^{T} \\
& +\sum_{i=0}^{2} \sum_{j=0}^{2} \alpha_{i} \Lambda_{i} \vec{C}_{s, k} N_{j} \Xi_{k} N_{j}^{T} \vec{C}_{s, k}^{T} \Lambda_{i}^{T},
\end{aligned}
$$


where

$$
\begin{aligned}
\mathscr{A}_{k}= & \alpha_{0} \alpha_{1} \Lambda_{0} \vec{C}_{k} \Xi_{k} \vec{C}_{k}^{T} \Lambda_{1}^{T}+\alpha_{0} \alpha_{2} \Lambda_{0} \vec{C}_{k} \Xi_{k} \vec{C}_{k}^{T} \Lambda_{2}^{T} \\
& +\alpha_{1} \alpha_{2} \Lambda_{1} \vec{C}_{k} \Xi_{k} \vec{C}_{k}^{T} \Lambda_{2}^{T} .
\end{aligned}
$$

Proof. By using Definition 3 and noting the expressions of $\vec{\Phi}_{k}$ and $\vec{H}_{k}$, one has

$$
\begin{aligned}
\Delta_{\vec{\Phi}_{k} \vec{\Phi}_{k}}\left(\Xi_{k}\right)= & \mathbb{E}\left\{\left[\vec{\Phi}_{k}-\mathbb{E}\left\{\vec{\Phi}_{k}\right\}\right] \vec{x}_{k} \vec{x}_{k}^{T}\left[\vec{\Phi}_{k}-\mathbb{E}\left\{\vec{\Phi}_{k}\right\}\right]^{T}\right\} \\
= & \mathbb{E}\left\{\xi_{k} \vec{A}_{s, k} \vec{x}_{k} \vec{x}_{k}^{T} \vec{A}_{s, k}^{T} \xi_{k}\right\} \\
= & \vec{A}_{s, k} \Xi_{k} \vec{A}_{s, k}^{T}, \\
\Delta_{\vec{H}_{k} \vec{H}_{k}}\left(\Xi_{k}\right)= & \mathbb{E}\left\{\left[\vec{H}_{k}-\bar{H}_{k}\right] \vec{x}_{k} \vec{x}_{k}^{T}\left[\vec{H}_{k}-\bar{H}_{k}\right]^{T}\right\} \\
= & \mathbb{E}\left\{\left[\left(\vec{\Lambda}_{k}-\bar{\Lambda}_{k}\right) \vec{C}_{k}+\vec{\Lambda}_{k} \vec{C}_{s, k} \vec{\eta}_{k}\right] \vec{x}_{k} \vec{x}_{k}^{T}\right. \\
& \left.\cdot\left[\left(\vec{\Lambda}_{k}-\bar{\Lambda}_{k}\right) \vec{C}_{k}+\vec{\Lambda}_{k} \vec{C}_{s, k} \vec{\eta}_{k}\right]^{T}\right\} \\
= & \mathbb{E}\left\{\left(\vec{\Lambda}_{k}-\bar{\Lambda}_{k}\right) \vec{C}_{k} \vec{x}_{k} \vec{x}_{k}^{T} \vec{C}_{k}^{T}\left(\vec{\Lambda}_{k}-\bar{\Lambda}_{k}\right)^{T}\right. \\
& +\vec{\Lambda}_{k} \vec{C}_{s, k} \vec{\eta}_{k} \vec{x}_{k} \vec{x}_{k}^{T} \vec{\eta}_{k} \vec{C}_{s, k}^{T} \vec{\Lambda}_{k}^{T} \\
& +\left(\vec{\Lambda}_{k}-\bar{\Lambda}_{k}\right) \vec{C}_{k} \vec{x}_{k} \vec{x}_{k}^{T} \vec{\eta}_{k} \vec{C}_{s, k}^{T} \vec{\Lambda}_{k}^{T} \\
& \left.+\vec{\Lambda}_{k} \vec{C}_{s, k} \vec{\eta}_{k} \vec{x}_{k} \vec{x}_{k}^{T} \vec{C}_{k}^{T}\left(\vec{\Lambda}_{k}-\bar{\Lambda}_{k}\right)^{T}\right\} \\
= & \alpha_{0}\left(1-\alpha_{0}\right) \Lambda_{0} \vec{C}_{k} \Xi_{k} \vec{C}_{k}^{T} \Lambda_{0}^{T} \\
& +\alpha_{1}\left(1-\alpha_{1}\right) \Lambda_{1} \vec{C}_{k} \Xi_{k} \vec{C}_{k}^{T} \Lambda_{1}^{T} \\
+ & \alpha_{2}\left(1-\alpha_{2}\right) \Lambda_{2} \vec{C}_{k} \Xi_{k} \vec{C}_{k}^{T} \Lambda_{2}^{T}-\mathscr{A}_{k}-\mathscr{A}_{k}^{T} \\
& +\sum_{i=0}^{2} \sum_{j=0}^{2} \alpha_{i} \Lambda_{i} \vec{C}_{s, k} N_{j} \Xi_{k} N_{j}^{T} \vec{C}_{s, k}^{T} \Lambda_{i}^{T},
\end{aligned}
$$

where $\mathscr{A}_{k}$ is defined in (15). Then, the proof of this lemma is complete.

Lemma 5. The state covariance matrix $\Xi_{k}$ of system (7) satisfies the following recursion:

$$
\Xi_{k}=\bar{\Phi}_{k-1} \Xi_{k-1} \bar{\Phi}_{k-1}^{T}+\Delta_{\vec{\Phi}_{k-1} \vec{\Phi}_{k-1}}\left(\Xi_{k-1}\right)+\vec{B}_{k-1} Q_{\omega_{k-1}} \vec{B}_{k-1}^{T}
$$

with the initial value $\Xi_{0}=\operatorname{diag}\left\{P_{0}+\mu_{0} \mu_{0}^{T}, 0,0\right\}$.

Proof. It follows from (7) that

$$
\begin{aligned}
\Xi_{k}= & \mathbb{E}\left\{\vec{x}_{k} \vec{x}_{k}^{T}\right\} \\
= & \mathbb{E}\left\{\left[\vec{\Phi}_{k-1} \vec{x}_{k-1}+\vec{B}_{k-1} \vec{\omega}_{k-1}\right]\right. \\
& \left.\cdot\left[\vec{\Phi}_{k-1} \vec{x}_{k-1}+\vec{B}_{k-1} \vec{\omega}_{k-1}\right]^{T}\right\} \\
= & \bar{\Phi}_{k-1} \Xi_{k-1} \bar{\Phi}_{k-1}^{T}+\Delta_{\vec{\Phi}_{k-1} \vec{\Phi}_{k-1}}\left(\Xi_{k-1}\right)+\vec{B}_{k-1} Q_{\omega_{k-1}} \vec{B}_{k-1}^{T} .
\end{aligned}
$$

The proof of this lemma is complete.
Now, we are ready to design the optimal Kalman filter for system (7)-(8) based on the observation sequence $\left\{y_{1}, y_{2}, \ldots, y_{k-1}\right\}$. By employing Lemmas 4 and 5 , we have the following theorem.

Theorem 6. The optimal Kalman filtering for system (7)-(8) is given as follows:

$$
\begin{aligned}
\hat{\vec{x}}_{k \mid k}= & \hat{\vec{x}}_{k \mid k-1}+K_{k} \varepsilon_{k} \\
\widehat{\vec{x}}_{k \mid k-1}= & \bar{\Phi}_{k-1} \widehat{\vec{x}}_{k-1 \mid k-1} \\
\varepsilon_{k}= & \vec{y}_{k}-\bar{H}_{k} \widehat{\vec{x}}_{k \mid k-1}-\bar{\Lambda}_{k}\left(F_{k-1} \varepsilon_{k-1}+G_{k-2} \varepsilon_{k-2}\right) \\
F_{k-1}= & {\left[\delta_{k-1}-G_{k-2}\left(K_{k-2}^{T} \bar{\Phi}_{k-2}^{T} \bar{H}_{k-1}^{T}+F_{k-2}^{T} \bar{\Lambda}_{k-1}^{T}\right)\right] Q_{\varepsilon_{k-1}}^{-1} } \\
G_{k-2}= & \mathcal{N}_{k-2} Q_{\varepsilon_{k-2}}^{-1} \\
P_{k \mid k}= & P_{k \mid k-1}-K_{k} Q_{\varepsilon_{k}} K_{k}^{T} \\
P_{k \mid k-1}= & \bar{\Phi}_{k-1} P_{k-1 \mid k-1} \bar{\Phi}_{k-1}^{T}+\Delta_{\vec{\Phi}_{k-1} \vec{\Phi}_{k-1}}\left(\Xi_{k-1}\right) \\
& +\vec{B}_{k-1} Q_{\omega_{k-1}} \vec{B}_{k-1}^{T}, \\
K_{k}= & {\left[P_{k \mid k-1} \bar{H}_{k}^{T}-\bar{\Phi}_{k-1} L_{k-2} Q_{\varepsilon_{k-2}} G_{k-2}^{T} \bar{\Lambda}_{k}^{T}\right.} \\
& \left.-L_{k-1} Q_{\varepsilon_{k-1}} F_{k-1}^{T} \bar{\Lambda}_{k}^{T}\right] Q_{\varepsilon_{k}}^{-1} \\
L_{k-1}= & {\left[\bar{\Phi}_{k-1} P_{k-1 \mid k-2} \bar{H}_{k-1}^{T}\right.} \\
& -\bar{\Phi}_{k-1} L_{k-2} Q_{\varepsilon_{k-2}}^{T} F_{k-2}^{T} \bar{\Lambda}_{k-1}^{T} \\
& \left.-\bar{\Phi}_{k-1} \bar{\Phi}_{k-2} L_{k-3} Q_{\varepsilon_{k-3}}^{T} G_{k-3}^{T} \bar{\Lambda}_{k-1}^{T}\right] Q_{\varepsilon_{k-1}}^{-1} \\
Q_{\varepsilon_{k}}= & \Delta_{H_{k} \vec{H}_{k}}\left(\Xi_{k}\right)+\bar{H}_{k} P_{k \mid k-1} \bar{H}_{k}^{T} \\
& +\sum_{i=0}^{2} \alpha_{i} \Lambda_{i} Q_{k} \Lambda_{i}^{T}+\bar{\Lambda}_{k} G_{k-2} Q_{\varepsilon_{k-2}} G_{k-2}^{T} \bar{\Lambda}_{k}^{T} \\
& +\bar{\Lambda}_{k} F_{k-1} Q_{\varepsilon_{k-1}} F_{k-1}^{T} \bar{\Lambda}_{k}^{T}+\mathscr{B}_{k}+\mathscr{B}_{k}^{T} \\
&
\end{aligned}
$$

where

$$
\begin{aligned}
& Q_{k}=\operatorname{diag}\left\{Q_{\gamma_{k}}, Q_{\gamma_{k-1}}, Q_{\gamma_{k-2}}\right\}, \\
& \mathcal{S}_{k-1}=\left[\begin{array}{llll}
0 & \alpha_{0} Q_{\gamma_{k-1}} & \alpha_{1} Q_{\gamma_{k-2}}
\end{array}\right]^{T}, \\
& \mathcal{N}_{k-2}=\left[\begin{array}{lll}
0 & 0 & \alpha_{0} Q_{v_{k-2}}
\end{array}\right]^{T}, \\
& \mathscr{B}_{k}=\bar{H}_{k}\left(\bar{\Phi}_{k-1} \bar{\Phi}_{k-2} K_{k-2} G_{k-2}^{T}+\bar{\Phi}_{k-1} K_{k-1} F_{k-1}^{T}\right) \bar{\Lambda}_{k}^{T} \\
& -\bar{\Lambda}_{k} G_{k-2} G_{k-2}^{T} \bar{\Lambda}_{k}^{T}-\bar{\Lambda}_{k} F_{k-1} F_{k-1}^{T} \bar{\Lambda}_{k}^{T} \text {. }
\end{aligned}
$$

Proof. According to the projection theory, it is easy to obtain (19). Moreover, the filter gain matrix $K_{k}$ is calculated by

$$
K_{k}=\mathbb{E}\left\{\vec{x}_{k} \varepsilon_{k}^{T}\right\}\left[\mathbb{E}\left\{\varepsilon_{k} \varepsilon_{k}^{T}\right\}\right]^{-1} .
$$

Taking projection on both sides of (7) onto the linear space spanned by $\left\{\vec{y}_{1}, \vec{y}_{2}, \ldots, \vec{y}_{k-1}\right\}$, we have

$$
\widehat{\vec{x}}_{k \mid k-1}=\bar{\Phi}_{k-1} \widehat{\vec{x}}_{k-1 \mid k-1}+\vec{B}_{k-1} \widehat{\vec{\omega}}_{k-1 \mid k-1} \text {. }
$$

From the projection theory, we have $\widehat{\vec{\omega}}_{k-1 \mid k-1}=0$. Then, (20) can be obtained directly. 
Set the innovation

$$
\varepsilon_{k}=\vec{y}_{k}-\widehat{\vec{y}}_{k \mid k-1} \text {. }
$$

Taking projection on both sides of (8) onto the linear space spanned by $\left\{\vec{y}_{1}, \vec{y}_{2}, \ldots, \vec{y}_{k-1}\right\}$, we have

$$
\widehat{\vec{y}}_{k \mid k-1}=\bar{H}_{k} \widehat{\vec{x}}_{k \mid k-1}+\bar{\Lambda}_{k} \widehat{\vec{\nu}}_{k \mid k-1}
$$

where the one-step prediction $\widehat{\vec{v}}_{k \mid k-1}$ of the measurement noise is calculated by

$$
\widehat{\vec{v}}_{k \mid k-1}=\widehat{\vec{v}}_{k \mid k-2}+F_{k-1} \varepsilon_{k-1} \text {. }
$$

Here, the one-step prediction gain $F_{k-1}$ of the measurement noise is defined by

$$
F_{k-1}=\mathbb{E}\left\{\vec{\nu}_{k} \varepsilon_{k-1}^{T}\right\}\left[\mathbb{E}\left\{\varepsilon_{k-1} \varepsilon_{k-1}^{T}\right\}\right]^{-1} .
$$

Moreover, the two-step prediction $\widehat{\vec{\nu}}_{k \mid k-2}$ of the measurement noise in (34) is computed by

$$
\widehat{\vec{v}}_{k \mid k-2}=\widehat{\vec{v}}_{k \mid k-3}+G_{k-2} \varepsilon_{k-2}
$$

where the two-step prediction gain of the measurement noise is defined by

$$
G_{k-2}=\mathbb{E}\left\{\vec{v}_{k} \varepsilon_{k-2}^{T}\right\}\left[\mathbb{E}\left\{\varepsilon_{k-2} \varepsilon_{k-2}^{T}\right\}\right]^{-1} .
$$

From the projection theory, $\widehat{\vec{v}}_{k} \perp \mathscr{L}\left\{\vec{y}_{1}, \vec{y}_{2}, \ldots, \vec{y}_{k-3}\right\}$, where the symbol $\perp$ denotes the orthogonality. Then, it is not difficult to see that $\widehat{\vec{v}}_{k \mid k-3}=0$. Subsequently, substituting (34) and (36) into (33) yields

$$
\widehat{\vec{y}}_{k \mid k-1}=\bar{H}_{k} \widehat{\vec{x}}_{k \mid k-1}+\bar{\Lambda}_{k}\left(F_{k-1} \varepsilon_{k-1}+G_{k-2} \varepsilon_{k-2}\right) \text {. }
$$

Then, it follows from (32) and (38) that (21) is true.

The innovation $\varepsilon_{k}$ can be rewritten as follows:

$$
\begin{aligned}
\varepsilon_{k}= & \vec{H}_{k} \vec{x}_{k}+\vec{\Lambda}_{k} \vec{v}_{k}-\bar{H}_{k} \widehat{\vec{x}}_{k \mid k-1} \\
& -\bar{\Lambda}_{k}\left(F_{k-1} \varepsilon_{k-1}+G_{k-2} \varepsilon_{k-2}\right) \\
= & \bar{H}_{k} \widetilde{\vec{x}}_{k \mid k-1}+\Delta H_{k} \vec{x}_{k}+\vec{\Lambda}_{k} \vec{v}_{k} \\
& -\bar{\Lambda}_{k}\left(F_{k-1} \varepsilon_{k-1}+G_{k-2} \varepsilon_{k-2}\right),
\end{aligned}
$$

where $\widetilde{\vec{x}}_{k \mid k-1}=\vec{x}_{k}-\widehat{\vec{x}}_{k \mid k-1}$ is the one-step prediction error. Substitute (39) with $k=k-1$ into (35). Noting

$$
\begin{aligned}
\widetilde{\vec{x}}_{k-1 \mid k-2} & =\vec{x}_{k-1}-\widehat{\vec{x}}_{k-1 \mid k-2} \\
& =\bar{\Phi}_{k-2} \widetilde{\vec{x}}_{k-2 \mid k-2}+\Delta \Phi_{k-2} \vec{x}_{k-2}+\vec{B}_{k-2} \vec{\omega}_{k-2}, \\
\widetilde{\vec{x}}_{k-2 \mid k-2} & =\widetilde{\vec{x}}_{k-2 \mid k-3}-K_{k-2} \varepsilon_{k-2},
\end{aligned}
$$

the one-step prediction gain $F_{k-1}$ of the measurement noise can be calculated

$$
\begin{gathered}
F_{k-1}=\mathbb{E}\left\{\vec{v}_{k} \varepsilon_{k-1}^{T}\right\}\left[\mathbb{E}\left\{\varepsilon_{k-1} \varepsilon_{k-1}^{T}\right\}\right]^{-1} \\
=\mathbb{E}\left\{\vec { v } _ { k } \left[\bar{H}_{k-1} \widetilde{\vec{x}}_{k-1 \mid k-2}+\Delta H_{k-1} \vec{x}_{k-1}+\vec{\Lambda}_{k-1} \vec{v}_{k-1}\right.\right. \\
\left.\left.-\bar{\Lambda}_{k-1}\left(F_{k-2} \varepsilon_{k-2}+G_{k-3} \varepsilon_{k-3}\right)\right]^{T}\right\} \\
\cdot\left[\mathbb{E}\left\{\varepsilon_{k-1} \varepsilon_{k-1}^{T}\right\}\right]^{-1} .
\end{gathered}
$$

When deriving (41), we have used the fact that $\vec{v}_{k} \perp \varepsilon_{k-3}$ and $\mathbb{E}\left\{\Delta H_{k-1}\right\}=0$. Then, we have (22). Similarly, substituting (39) with $k=k-2$ into (37), one has (23).

Subsequently, we are in a position to obtain the filtering error covariance matrix $P_{k \mid k}$ and the prediction error covariance matrix $P_{k \mid k-1}$. Subtracting (19) from $\vec{x}_{k \mid k}$, the filtering error equation can be obtained:

$$
\widetilde{\vec{x}}_{k \mid k}=\vec{x}_{k}-\widehat{\vec{x}}_{k \mid k}=\widetilde{\vec{x}}_{k \mid k-1}-K_{k} \varepsilon_{k} .
$$

Then, we have

$$
\widetilde{\vec{x}}_{k \mid k}+K_{k} \varepsilon_{k}=\widetilde{\vec{x}}_{k \mid k-1} .
$$

Notice that $\widetilde{\vec{x}}_{k \mid k} \perp \varepsilon_{k}$, $\mathbb{E}\left\{\Delta \Phi_{k-1}\right\}=0, \vec{x}_{k-1}$, and $\widetilde{\vec{x}}_{k-1 \mid k-1}$ are all uncorrelated with $\vec{\omega}_{k-1}$, we have

$$
\begin{aligned}
P_{k \mid k} & =\mathbb{E}\left\{\widetilde{\vec{x}}_{k \mid k} \widetilde{\vec{x}}_{k \mid k}^{T}\right\} \\
& =\mathbb{E}\left\{\left[\widetilde{\vec{x}}_{k \mid k}+K_{k} \varepsilon_{k}-K_{k} \varepsilon_{k}\right]\left[\widetilde{\vec{x}}_{k \mid k}+K_{k} \varepsilon_{k}-K_{k} \varepsilon_{k}\right]^{T}\right\} \\
& =P_{k \mid k-1}-K_{k} Q_{\varepsilon_{k}} K_{k}^{T} .
\end{aligned}
$$

Thus, (24) is obtained.

Similarly, the one-step prediction error equation can be obtained as follows:

$$
\begin{aligned}
\widetilde{\vec{x}}_{k \mid k-1}= & \vec{x}_{k}-\widehat{\vec{x}}_{k \mid k-1} \\
= & \vec{\Phi}_{k-1} \vec{x}_{k-1}+\vec{B}_{k-1} \vec{\omega}_{k-1}-\bar{\Phi}_{k-1} \widehat{\vec{x}}_{k-1 \mid k-1} \\
= & \left(\bar{\Phi}_{k-1}+\Delta \Phi_{k-1}\right) \vec{x}_{k-1}+\vec{B}_{k-1} \vec{\omega}_{k-1} \\
& -\bar{\Phi}_{k-1} \widehat{\vec{x}}_{k-1 \mid k-1} \\
= & \bar{\Phi}_{k-1} \widetilde{\vec{x}}_{k-1 \mid k-1}+\Delta \Phi_{k-1} \vec{x}_{k-1}+\vec{B}_{k-1} \vec{\omega}_{k-1} .
\end{aligned}
$$

According to (45), we have the following equation:

$$
\begin{aligned}
P_{k \mid k-1}= & \mathbb{E}\left\{\widetilde{\vec{x}}_{k \mid k-1} \widetilde{\vec{x}}_{k \mid k-1}^{T}\right\} \\
= & \bar{\Phi}_{k-1} P_{k-1 \mid k-1} \bar{\Phi}_{k-1}^{T} \\
& +\Delta_{\vec{\Phi}_{k-1} \vec{\Phi}_{k-1}}\left(\Xi_{k-1}\right)+\vec{B}_{k-1} Q_{\omega_{k-1}} \vec{B}_{k-1}^{T} \\
& +\mathscr{C}_{k-1}+\mathscr{C}_{k-1}^{T},
\end{aligned}
$$


where

$$
\begin{aligned}
\mathscr{C}_{k-1}= & \bar{\Phi}_{k-1} \mathbb{E}\left\{\widetilde{\vec{x}}_{k-1 \mid k-1} \vec{x}_{k-1}^{T} \Delta \Phi_{k-1}^{T}\right\} \\
& +\bar{\Phi}_{k-1} \mathbb{E}\left\{\widetilde{\vec{x}}_{k-1 \mid k-1} \vec{\omega}_{k-1}^{T}\right\} \vec{B}_{k-1}^{T} \\
& +\mathbb{E}\left\{\Delta \Phi_{k-1} \vec{x}_{k-1} \vec{\omega}_{k-1}^{T}\right\} \vec{B}_{k-1}^{T} .
\end{aligned}
$$

Noting $\mathscr{C}_{k-1}=0$, we have

$$
\begin{aligned}
P_{k \mid k-1}= & \bar{\Phi}_{k-1} P_{k-1 \mid k-1} \bar{\Phi}_{k-1}^{T} \\
& +\Delta_{\vec{\Phi}_{k-1} \vec{\Phi}_{k-1}}\left(\Xi_{k-1}\right)+\vec{B}_{k-1} Q_{\omega_{k-1}} \vec{B}_{k-1}^{T} .
\end{aligned}
$$

Then, it is concluded that (25) holds.

Next, we aim to derive the filter gain $K_{k}$. Firstly, substitute (39) into (30). Secondly, by using $\mathbb{E}\left\{\Delta H_{k}\right\}=0$ and $\vec{x}_{k}=$ $\widehat{\vec{x}}_{k \mid k-1}+\widetilde{\vec{x}}_{k \mid k-1}$, we obtain

$$
\begin{aligned}
K_{k}= & \mathbb{E}\left\{\vec{x}_{k} \varepsilon_{k}^{T}\right\} Q_{\varepsilon_{k}}^{-1} \\
= & \mathbb{E}\left\{\vec { x } _ { k } \left[\Delta H_{k} \vec{x}_{k}+\bar{H}_{k} \widetilde{\vec{x}}_{k \mid k-1}+\vec{\Lambda}_{k} \vec{v}_{k}\right.\right. \\
& \left.\left.-\bar{\Lambda}_{k}\left(F_{k-1} \varepsilon_{k-1}+G_{k-2} \varepsilon_{k-2}\right)\right]^{T}\right\} Q_{\varepsilon_{k}}^{-1} \\
= & \left\{P_{k \mid k-1} \bar{H}_{k}^{T}+\mathbb{E}\left\{\vec{x}_{k} \vec{v}_{k}^{T} \vec{\Lambda}_{k}^{T}\right\}-\mathscr{M}_{k}\right\} Q_{\varepsilon_{k}}^{-1},
\end{aligned}
$$

where $\mathscr{M}_{k}=\mathbb{E}\left\{\vec{x}_{k}\left(F_{k-1} \varepsilon_{k-1}+G_{k-2} \varepsilon_{k-2}\right)^{T} \bar{\Lambda}_{k}^{T}\right\}$. When deriving (49), we have used the fact that $\vec{x}_{k}$ is uncorrelated with $\vec{v}_{k}$. Setting

$$
\mathbb{E}\left\{\vec{x}_{k} \varepsilon_{k-1}^{T}\right\} Q_{\varepsilon_{k-1}}^{-1}=L_{k-1},
$$

we have $\mathbb{E}\left\{\vec{x}_{k} \varepsilon_{k-1}^{T}\right\}=L_{k-1} Q_{\varepsilon_{k-1}}$. By using (7) and noting $\vec{\omega}_{k-1} \perp \varepsilon_{k-2}$, the term $\mathscr{M}_{k}$ can be obtained as follows:

$$
\begin{aligned}
\mathscr{M}_{k}= & \mathbb{E}\left\{\vec{x}_{k} \varepsilon_{k-1}^{T}\right\} F_{k-1}^{T} \bar{\Lambda}_{k}^{T}+\mathbb{E}\left\{\vec{x}_{k} \varepsilon_{k-2}^{T}\right\} G_{k-2}^{T} \bar{\Lambda}_{k}^{T} \\
= & \mathbb{E}\left\{\vec{x}_{k} \varepsilon_{k-1}^{T}\right\} F_{k-1}^{T} \bar{\Lambda}_{k}^{T} \\
& +\mathbb{E}\left\{\left(\vec{\Phi}_{k-1} \vec{x}_{k-1}+\vec{B}_{k-1} \vec{\omega}_{k-1}\right) \varepsilon_{k-2}^{T}\right\} G_{k-2}^{T} \bar{\Lambda}_{k}^{T} \\
= & L_{k-1} Q_{\varepsilon_{k-1}} F_{k-1}^{T} \bar{\Lambda}_{k}^{T}+\bar{\Phi}_{k-1} L_{k-2} Q_{\varepsilon_{k-2}} G_{k-2}^{T} \bar{\Lambda}_{k}^{T} .
\end{aligned}
$$

Substituting (51) into (49) and noting $\mathbb{E}\left\{\vec{x}_{k} \vec{v}_{k}^{T} \vec{\Lambda}_{k}^{T}\right\}=0$, we have (26). that

Furthermore, it follows from $\vec{x}_{k-1}=\widehat{\vec{x}}_{k-1 \mid k-2}+\widetilde{\vec{x}}_{k-1 \mid k-2}$

$$
\begin{aligned}
\mathbb{E}\left\{\vec{x}_{k} \varepsilon_{k-1}^{T}\right\}= & \mathbb{E}\left\{\vec { x } _ { k } \left[\bar{H}_{k-1} \widetilde{\vec{x}}_{k-1 \mid k-2}+\Delta H_{k-1} \vec{x}_{k-1}+\vec{\Lambda}_{k-1} \vec{v}_{k-1}\right.\right. \\
& \left.\left.-\bar{\Lambda}_{k-1}\left(F_{k-2} \varepsilon_{k-2}+G_{k-3} \varepsilon_{k-3}\right)\right]^{T}\right\} \\
= & \bar{\Phi}_{k-1} P_{k-1 \mid k-2} \bar{H}_{k-1}^{T}-\bar{\Phi}_{k-1} L_{k-2} Q_{\varepsilon_{k-2}}^{T} F_{k-2}^{T} \bar{\Lambda}_{k-1}^{T} \\
& -\bar{\Phi}_{k-1} \bar{\Phi}_{k-2} L_{k-3} Q_{\varepsilon_{k-3}}^{T} G_{k-3}^{T} \bar{\Lambda}_{k-1}^{T}
\end{aligned}
$$

Substituting (52) into (50), we can see that (27) is true.
Finally, we will derive the term $Q_{\varepsilon_{k}}$ in (28). According to (39), we have

$$
\begin{aligned}
& Q_{\varepsilon_{k}}=\mathbb{E}\left\{\varepsilon_{k} \varepsilon_{k}^{T}\right\} \\
&=\mathbb{E}\{ {\left[\Delta H_{k} \vec{x}_{k}+\bar{H}_{k} \widetilde{\vec{x}}_{k \mid k-1}+\vec{\Lambda}_{k} \vec{v}_{k}\right.} \\
&\left.\quad \bar{\Lambda}_{k}\left(F_{k-1} \varepsilon_{k-1}+G_{k-2} \varepsilon_{k-2}\right)\right] \\
& \times\left[\Delta H_{k} \vec{x}_{k}+\bar{H}_{k} \widetilde{\vec{x}}_{k \mid k-1}+\vec{\Lambda}_{k} \vec{v}_{k}\right. \\
&\left.\left.-\bar{\Lambda}_{k}\left(F_{k-1} \varepsilon_{k-1}+G_{k-2} \varepsilon_{k-2}\right)\right]^{T}\right\} \\
&=\mathbb{E}\left\{\Delta H_{k} \vec{x}_{k} \vec{x}_{k}^{T} \Delta H_{k}^{T}\right\} \\
&+ \mathbb{E}\left\{\bar{H}_{k} \widetilde{\vec{x}}_{k \mid k-1} \widetilde{\vec{x}}_{k \mid k-1}^{T} \bar{H}_{k}^{T}\right\}+\mathbb{E}\left\{\vec{\Lambda}_{k} \vec{v}_{k} \vec{v}_{k}^{T} \vec{\Lambda}_{k}^{T}\right\} \\
&+ \mathbb{E}\left\{\bar{\Lambda}_{k}\left[F_{k-1} \varepsilon_{k-1}+G_{k-2} \varepsilon_{k-2}\right]\right. \\
&=\left.\quad\left[F_{k-1} \varepsilon_{k-1}+G_{k-2} \varepsilon_{k-2}\right]^{T} \bar{\Lambda}_{k}^{T}\right\}+\mathscr{D}_{k}+\mathscr{D}_{k}^{T} \\
& \Delta_{\vec{H}_{k} \vec{H}_{k}}\left(\Xi_{k}\right)+\bar{H}_{k} P_{k \mid k-1} \bar{H}_{k}^{T} \\
&+\sum_{i=0}^{2} \alpha_{i} \Lambda_{i} Q_{k} \Lambda_{i}^{T}+\bar{\Lambda}_{k} G_{k-2} Q_{\varepsilon_{k-2}} G_{k-2}^{T} \bar{\Lambda}_{\mathrm{k}}^{T} \\
&+ \bar{\Lambda}_{k} F_{k-1} Q_{\varepsilon_{k-1}} F_{k-1}^{T} \bar{\Lambda}_{k}^{T}+\mathscr{B}_{k}+\mathscr{B}_{k}^{T},
\end{aligned}
$$

where

$$
\begin{aligned}
\mathscr{D}_{k}=\mathbb{E}\{\Delta & H_{k} \vec{x}_{k} \widetilde{\vec{x}}_{k \mid k-1}^{T} \bar{H}_{k}^{T}+\Delta H_{k} \vec{x}_{k} \vec{v}_{k-1}^{T} \vec{\Lambda}_{k}^{T} \\
& -\Delta H_{k} \vec{x}_{k}\left[F_{k-1} \varepsilon_{k-1}+G_{k-2} \varepsilon_{k-2}\right]^{T} \bar{\Lambda}_{k}^{T} \\
& +\bar{H}_{k} \widetilde{\vec{x}}_{k \mid k-1} \vec{v}_{k}^{T} \vec{\Lambda}_{k}^{T} \\
& -\bar{H}_{k} \widetilde{\vec{x}}_{k \mid k-1}\left[F_{k-1} \varepsilon_{k-1}+G_{k-2} \varepsilon_{k-2}\right]^{T} \bar{\Lambda}_{k-1}^{T} \\
& \left.-\vec{\Lambda}_{k} \vec{v}_{k-1}\left[F_{k-1} \varepsilon_{k-1}+G_{k-2} \varepsilon_{k-2}\right]^{T} \bar{\Lambda}_{k}^{T}\right\},
\end{aligned}
$$

$Q_{k}$ and $\mathscr{B}_{k}$ are defined in (29). When deriving (53), we have used the fact that $\mathbb{E}\left\{\Delta H_{k}\right\}=0, \mathbb{E}\left\{\vec{x}_{k} \vec{v}_{k}^{T}\right\}=0$, and $\Delta H_{k}$ is uncorrelated with $\vec{x}_{k} \hat{\vec{x}}_{k \mid k-1}^{T}$. Up to now, the proof of Theorem 6 is complete.

So far, we have derived the Kalman filtering for the addressed linear stochastic systems with multiplicative noises and random two-step sensor delays. In the following, let us discuss the initial time instant.

Particularly, when $k=1$, (3) becomes $y_{1}=z_{1}$. In the augmented system (7)-(8), letting $\gamma_{k}^{2}=\gamma_{k}^{1}=0$, we have

$$
\begin{gathered}
\vec{\Lambda}_{1}=\left[\begin{array}{lll}
I & 0 & 0
\end{array}\right], \\
\Delta_{\vec{H}_{1} \vec{H}_{1}}\left(\Xi_{1}\right)=\sum_{j=0}^{2} \Lambda_{0} \vec{C}_{s, 1} N_{j} \Xi_{1} N_{j}^{T} \vec{C}_{s, 1}^{T} \Lambda_{0}^{T}, \\
F_{0}=\left\{\mathcal{S}_{0}-G_{-1}\left(K_{-1}^{T} \bar{\Phi}_{-1}^{T} \vec{H}_{0}^{T}+F_{-1}^{T} \bar{\Lambda}_{0}^{T}\right)\right\} Q_{\varepsilon_{0}}^{-1}, \\
G_{-1}=\mathcal{N}_{-1} Q_{\varepsilon_{-1}}^{-1},
\end{gathered}
$$




$$
\begin{aligned}
Q_{\varepsilon_{1}}= & \Delta_{\vec{H}_{1} \vec{H}_{1}}\left(\Xi_{1}\right)+\bar{H}_{1} P_{1 \mid 0} \bar{H}_{1}^{T}+\Lambda_{0} Q_{1} \Lambda_{0}^{T} \\
& +\bar{\Lambda}_{1} G_{-1} Q_{\varepsilon_{-1}} G_{-1}^{T} \bar{\Lambda}_{1}^{T}+\bar{\Lambda}_{1} F_{0} Q_{\varepsilon_{0}} F_{0}^{T} \bar{\Lambda}_{1}^{T}+\mathscr{B}_{1}+\mathscr{B}_{1}^{T},
\end{aligned}
$$

where

$$
\mathcal{S}_{0}=\left[\begin{array}{lll}
0 & 0 & 0
\end{array}\right]^{T}, \quad \mathcal{N}_{-1}=\left[\begin{array}{lll}
0 & 0 & 0
\end{array}\right]^{T},
$$

and $\mathscr{B}_{1}$ is defined in (29).

Similarly, when $k=2$, (3) becomes $y_{2}=\gamma_{2}^{0} z_{2}+\gamma_{2}^{1} z_{1}$. In the augmented system (7)-(8), letting $\gamma_{k}^{2}=0$, one has

$$
\begin{aligned}
& \vec{\Lambda}_{2}=\left[\begin{array}{lll}
\gamma_{2}^{0} I & \gamma_{2}^{1} I & 0
\end{array}\right], \\
& \Delta_{\vec{H}_{2} \vec{H}_{2}}\left(\Xi_{2}\right)=\sum_{i=0}^{1} \alpha_{i}\left(1-\alpha_{i}\right) \Lambda_{i} \vec{C}_{2} \Xi_{2} \vec{C}_{2}^{T} \Lambda_{i}^{T}-\mathscr{A}_{2}-\mathscr{A}_{2}^{T} \\
& +\sum_{i=0}^{1} \sum_{j=0}^{2} \alpha_{i} \Lambda_{i} \vec{C}_{s, 2} N_{j} \Xi_{2} N_{j}^{T} \vec{C}_{s, 2}^{T} \Lambda_{i}^{T}, \\
& F_{1}=\left\{\mathcal{S}_{1}-G_{0}\left(K_{0}^{T} \bar{\Phi}_{0}^{T} \bar{H}_{1}^{T}+F_{0}^{T} \bar{\Lambda}_{1}^{T}\right)\right\} Q_{\varepsilon_{1}}^{-1}, \\
& G_{0}=\mathcal{N}_{0} Q_{\varepsilon_{0}}^{-1}, \\
& Q_{\varepsilon_{2}}=\Delta_{\vec{H}_{2} \vec{H}_{2}}\left(\Xi_{2}\right)+\bar{H}_{2} P_{2 \mid 1} \bar{H}_{2}^{T}+\alpha_{0} \Lambda_{0} Q_{2} \Lambda_{0}^{T} \\
& +\alpha_{1} \Lambda_{1} Q_{2} \Lambda_{1}^{T}+\bar{\Lambda}_{2} G_{0} Q_{\varepsilon_{0}} G_{0}^{T} \bar{\Lambda}_{2}^{T} \\
& +\bar{\Lambda}_{2} F_{1} Q_{\varepsilon_{1}} F_{1}^{T} \bar{\Lambda}_{2}^{T}+\mathscr{B}_{2}+\mathscr{B}_{2}^{T},
\end{aligned}
$$

where

$$
\begin{gathered}
\mathcal{S}_{1}=\left[\begin{array}{lll}
0 & Q_{\gamma_{1}} & 0
\end{array}\right]^{T}, \quad \mathcal{N}_{0}=\left[\begin{array}{lll}
0 & 0 & 0
\end{array}\right]^{T}, \\
\mathscr{A}_{2}=\alpha_{0} \alpha_{1} \Lambda_{0} \vec{C}_{2} \Xi_{2} \vec{C}_{2}^{T} \Lambda_{1}^{T},
\end{gathered}
$$

and $\mathscr{B}_{2}$ is defined in (29).

Remark 7. It is worth mentioning that when $\alpha_{0}=1$ and $A_{s, k}=C_{s, k}=0$, the developed optimal filtering is reduced to the traditional Kalman filtering algorithm. On the other hand, when $\alpha_{1}=1$ and $A_{s, k}=C_{s, k}=0$, the proposed filtering algorithm is the optimal Kalman filtering with onestep sensor delay.

To help understand, the calculation process of the proposed optimal Kalman filtering scheme in Theorem 6 can be summarized as follows.

Algorithm 8 (Kalman filtering with multiplicative noises and random two-step sensor delays).

Step 1. Give the initial values $\varepsilon_{-1}, \varepsilon_{0}, Q_{\varepsilon_{-2}}, Q_{\varepsilon_{-1}}, Q_{\varepsilon_{0}}, L_{-2}, L_{-1}$, $K_{-1}, K_{0}, F_{-1}, G_{-2}, P_{0 \mid 0}, P_{0 \mid-1}, \vec{x}_{0}, \widehat{\vec{x}}_{0}$, and $y_{1}$.

Step 2. Compute $\widehat{\vec{x}}_{1 \mid 0} \Rightarrow G_{-1} \Rightarrow F_{0} \Rightarrow \varepsilon_{1} \Rightarrow \Delta_{\vec{\Phi}_{0} \vec{\Phi}_{0}}\left(\Xi_{0}\right) \Rightarrow$ $\Xi_{1} \Rightarrow \Delta_{\vec{H}_{1} \vec{H}_{1}}\left(\Xi_{1}\right) \Rightarrow P_{1 \mid 0} \Rightarrow Q_{\varepsilon_{1}} \Rightarrow L_{0} \Rightarrow K_{1} \Rightarrow \widehat{\vec{x}}_{1 \mid 1} \Rightarrow P_{1 \mid 1}$ in turn.

Step 3. When $y_{2}$ is obtained, compute $\widehat{\vec{x}}_{2 \mid 1} \Rightarrow G_{0} \Rightarrow F_{1} \Rightarrow$ $\varepsilon_{2} \Rightarrow \Delta_{\vec{\Phi}_{1} \vec{\Phi}_{1}}\left(\Xi_{1}\right) \Rightarrow \Xi_{2} \Rightarrow \Delta_{\vec{H}_{2} \vec{H}_{2}}\left(\Xi_{2}\right) \Rightarrow P_{2 \mid 1} \Rightarrow Q_{\varepsilon_{2}} \Rightarrow$ $L_{1} \Rightarrow K_{2} \Rightarrow \widehat{\vec{x}}_{2 \mid 2} \Rightarrow P_{2 \mid 2}$ in turn.
Step 4. In general, calculate $\bar{x}_{k \mid k-1}$ by (20).

Step 5. Compute $G_{k-2}$ by (23). Substituting (23) into (22), we obtain $F_{k-1}$. Then, we can obtain $\varepsilon_{k}$ by substituting (22) and (23) into (21).

Step 6. Calculate $\Delta_{\vec{\Phi}_{k-1} \vec{\Phi}_{k-1}}\left(\Xi_{k-1}\right)$ by (13) and compute $\Xi_{k}$ by (17). By substituting $\Xi_{k}$ into (14), we have $\Delta_{\vec{H}_{k} \vec{H}_{k}}\left(\Xi_{k}\right)$.

Step 7. Calculate $P_{k \mid k-1}$ by substituting (13) into (25).

Step 8. Substituting (14), (22), (23), and (25) into (28), we obtain $Q_{\varepsilon_{k}}$.

Step 9. Compute $L_{k-1}$ by substituting (22), (23), (25), and (28) into (27).

Step 10. Substituting (25), (27), and (28) into (26), we obtain $K_{k}$.

Step 11. By using (19) and (24), we calculate the optimal estimation $\widehat{\vec{x}}_{k \mid k}$ and obtain $P_{k \mid k}$. Then, letting $k-1=k$, go back to Step 4 .

Remark 9. In this paper, we have used the state augmentation approach and innovation analysis technique to design the optimal Kalman filter contaminated with multiplicative noises and randomly occurring two-step sensor delays. Compared with the existing results, these two phenomena addressed have constituted the main differences and have been explicitly reflected in the main results, such as the terms $Q_{\varepsilon_{k}}, Q_{\varepsilon_{k-1}}, Q_{\varepsilon_{k-2}}$, and $Q_{\varepsilon_{k-3}}$. During the implementation of the proposed filtering algorithm, it is worth mentioning that more efforts should be made to derive the terms $L_{k-1}$ and $Q_{\varepsilon_{k}}$ in (27) and (28) due to the consideration of the randomly occurring sensor delays. From the above algorithm, it is easy to see that Steps 5-10 in Algorithm 8 are important especially those involved terms.

\section{An Illustrative Example}

In this section, a numerical example is proposed to show the feasibility and effectiveness of the proposed main results.

Consider the following system:

$$
\begin{gathered}
x_{k+1}=\left(\left[\begin{array}{cc}
0.2 & -0.15 \\
0 & 0.15
\end{array}\right]+\left[\begin{array}{cc}
0.01 & 0 \\
0 & 0.01
\end{array}\right] \xi_{k}\right) x_{k}+\left[\begin{array}{c}
2 \\
2.5
\end{array}\right] \omega_{k} \\
z_{k}=\left(\left[\begin{array}{cc}
1.5 & 1
\end{array}\right]+\left[\begin{array}{ll}
0.01 & 0.01
\end{array}\right] \eta_{k}\right) x_{k}+v_{k} \\
y_{k}=\sum_{i=0}^{\min \{k-1,2\}} \gamma_{k}^{i} z_{k-i},
\end{gathered}
$$

where $x_{k}=\left[\begin{array}{ll}x_{k, 1} & x_{k, 2}\end{array}\right]^{T}$ is the system state and $\omega_{k} \in \mathscr{R}$ and $v_{k} \in \mathscr{R}$ are uncorrelated white noises with zero means and variances $Q_{\omega_{k}}=0.1$ and $Q_{\nu_{k}}=0.2$, respectively. 
TABLE 1: Filter gains $K_{k}$ (Case I).

\begin{tabular}{|c|c|c|c|c|c|}
\hline$k$ & 1 & 2 & 3 & 4 & $\ldots$ \\
\hline \multirow{6}{*}{$K_{k}$} & 0.3527 & 0.3072 & 0.2391 & {$[0.2202]$} & \\
\hline & 0.4107 & 0.3822 & 0.3037 & 0.2853 & \\
\hline & 0.0903 & 0.0042 & 0.0293 & 0.0558 & : \\
\hline & -0.0226 & -0.0021 & 0.0360 & 0.0712 & \\
\hline & 0 & 0.0104 & 0.0008 & 0.0096 & \\
\hline & 0 & $-0.0141]$ & -0.0012 & {$[0.0117$} & \\
\hline
\end{tabular}

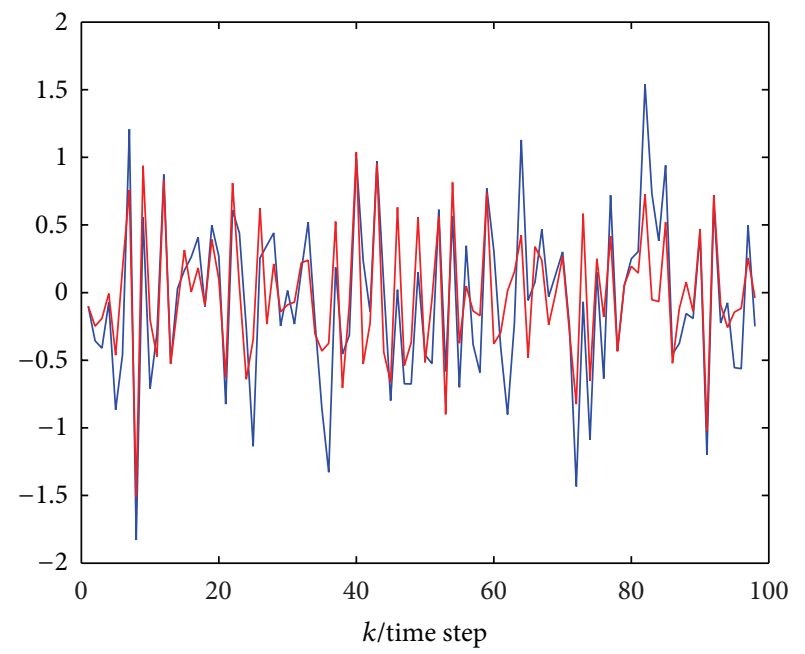

- Actual state $x_{k, 1}$

_ Filtering in this paper

FIGURE 1: The trajectories of $x_{k, 1}$ and $\widehat{x}_{k \mid k, 1}$ (Case I).

Let

$$
\begin{gathered}
\varepsilon_{-1}=1, \quad \varepsilon_{0}=1, \quad Q_{\varepsilon_{-2}}=1, \\
Q_{\varepsilon_{-1}}=1, \quad Q_{\varepsilon_{0}}=1, \quad P_{0 \mid 0}=I_{6 \times 6}, \\
P_{0 \mid-1}=I_{6 \times 6}, \\
L_{-2}=\left[\begin{array}{llllll}
0 & 0 & 0 & 0 & 0.1 & 0.2
\end{array}\right]^{T}, \\
L_{-1}=\left[\begin{array}{llllll}
0 & 0 & 0 & 0 & 0.2 & 0.3
\end{array}\right]^{T}, \\
F_{-1}=\left[\begin{array}{llllll}
0 & 0 & 0.1
\end{array}\right]^{T}, \\
\widehat{\vec{x}}_{0}=\left[\begin{array}{llllll}
-0.1 & 0.3 & 0 & 0 & 0 & 0
\end{array}\right]^{T}, \\
\vec{x}_{0}=\left[\begin{array}{llllll}
-0.1 & 0.3 & 0 & 0 & 0 & 0
\end{array}\right]^{T}, \\
K_{-1}=\left[\begin{array}{llllll}
0 & 0 & 0 & 0 & 0.4 & 0.2
\end{array}\right], \\
K_{0}=\left[\begin{array}{lllllll}
0 & 0 & 0 & 0 & 0 & 0.1
\end{array}\right] .
\end{gathered}
$$

According to Theorem 6, the optimal recursive filter $\widehat{x}_{k \mid k}$ can be obtained. The values of the filter gains are given as in Table 1. The trajectories of the actual states $x_{k, i}$ and their estimates $\widehat{x}_{k, i}(i=1,2)$ are plotted in Figures 1 and 2 . Let MSE $i$ denote the mean square error for the estimations of

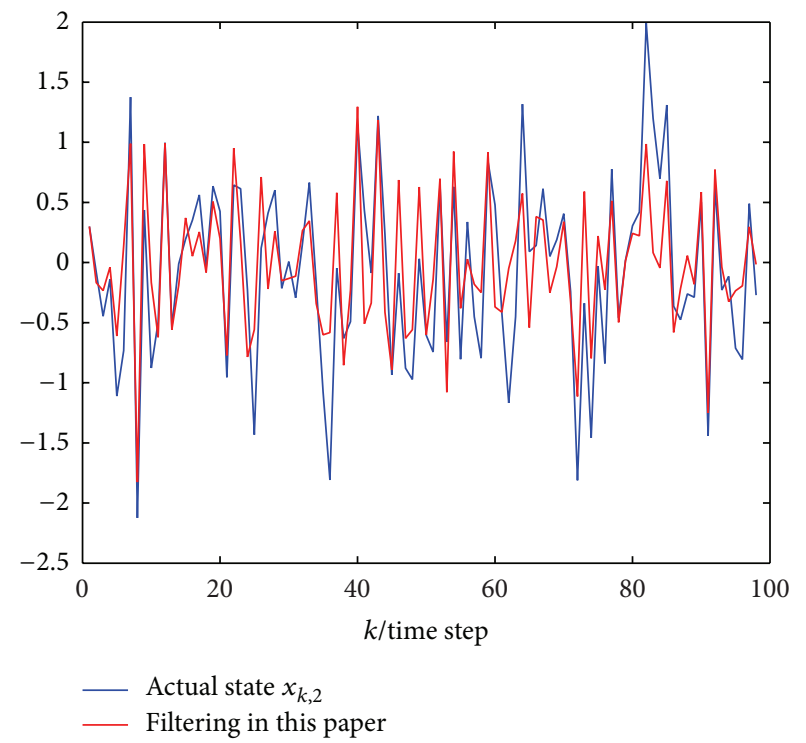

FIGURE 2: The trajectories of $x_{k, 2}$ and $\widehat{x}_{k \mid k, 2}$ (Case I).

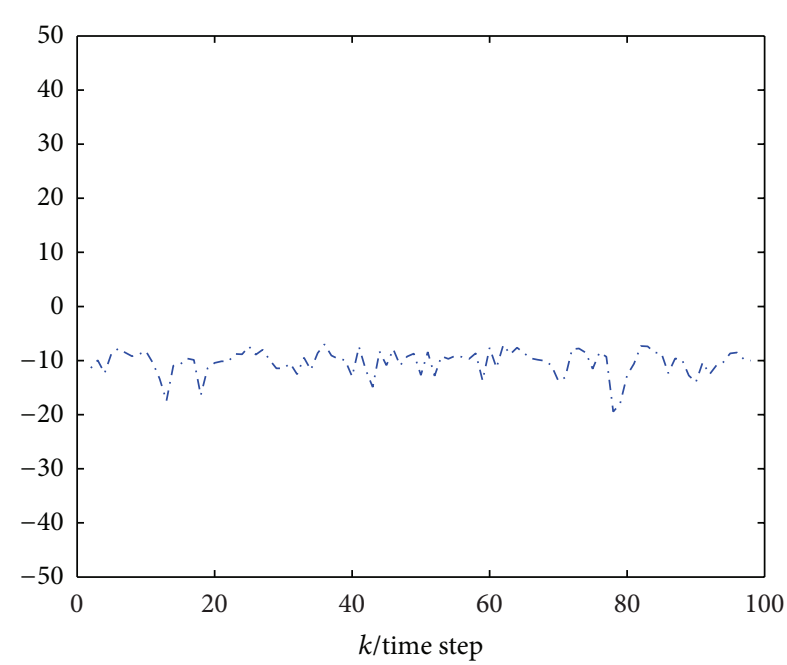

Figure 3: $\log (\mathrm{MSE1})$ (Case I).

$x_{k, i}$ and $x_{k, 2}$; that is, MSE $i=(1 / M) \sum_{j=1}^{M}\left(x_{k, i}-\widehat{x}_{k \mid k, i}\right)^{2}(i=$ $1,2)$, where $M$ is the number of simulation tests. Then, the $\log (\mathrm{MSE} i)(i=1,2)$ of the proposed filtering algorithm are plotted in Figures 3 and 4. 


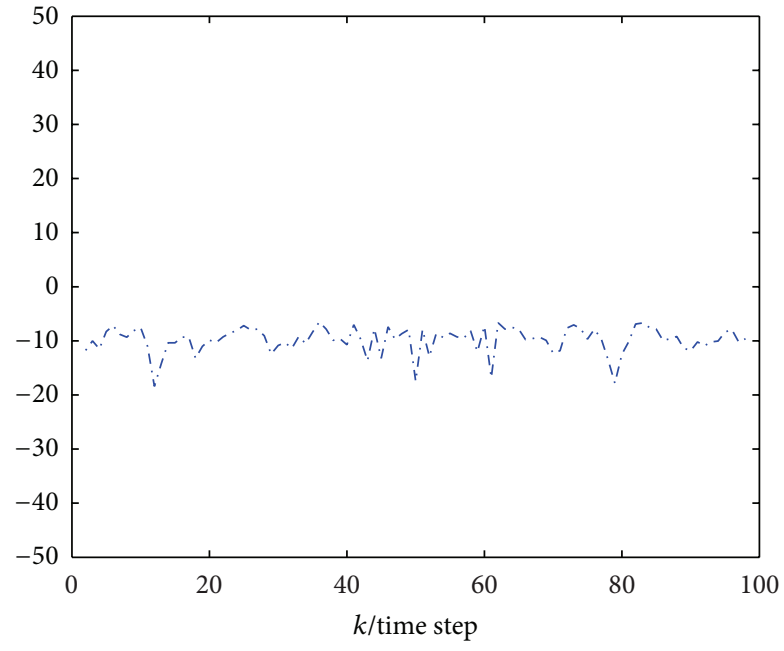

Figure 4: $\log (\mathrm{MSE} 2)$ (Case I).

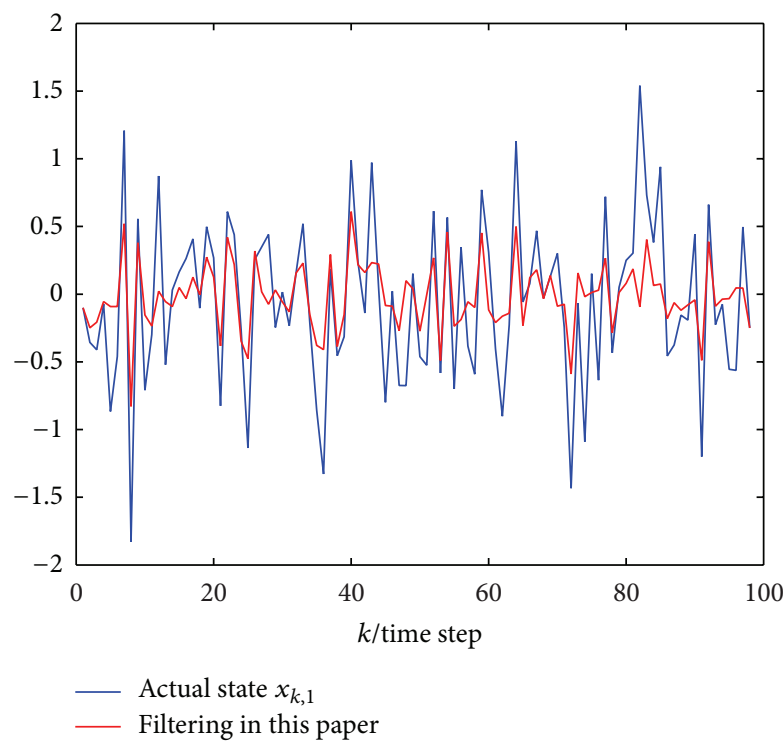

FIGURE 5: The trajectories of $x_{k, 1}$ and $\widehat{x}_{k \mid k, 1}$ (Case II).

In order to further discuss the effects from the randomly occurring two-step sensor delays, we make the comparison where the different probabilities of the sensor delays (i.e., Case I: $\alpha_{0}=0.95, \alpha_{1}=0.9$, and $\alpha_{2}=0.8$; Case II: $\alpha_{0}=0.65, \alpha_{1}=0.6$, and $\alpha_{2}=0.55$ ) are considered. The corresponding simulations are given in Figures 5-8. According to the simulations, we can see that the filtering performance is indeed influenced by the probabilities of the sensor delays. From the simulations, we can conclude that the developed filtering scheme performs well to estimate the addressed system with multiplicative noises and random twostep sensor delays. The reason is that we have made additional efforts during the algorithm design to attenuate the effects from the multiplicative noises and randomly occurring twostep sensor delays.

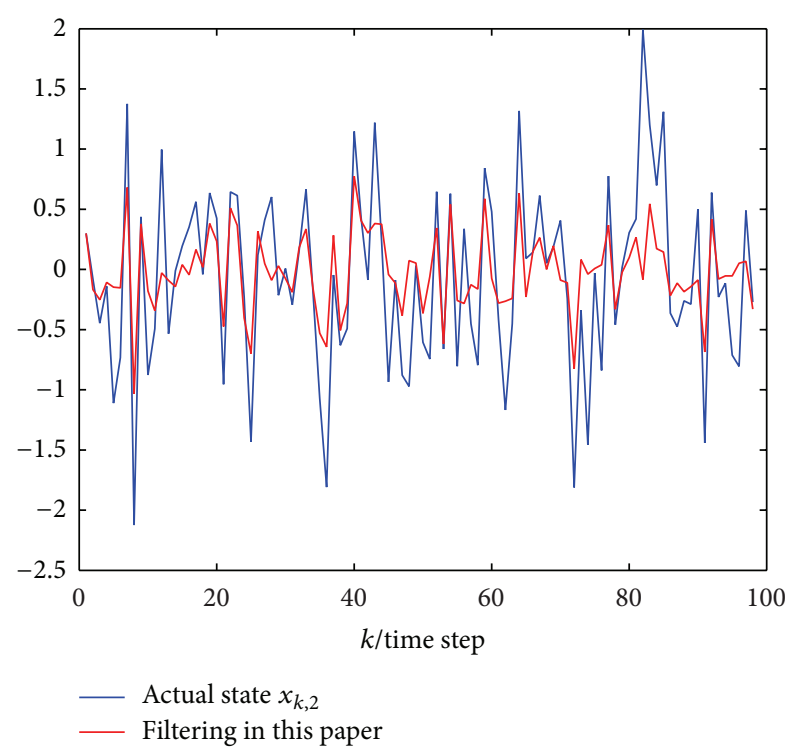

FIGURE 6: The trajectories of $x_{k, 2}$ and $\widehat{x}_{k \mid k, 2}$ (Case II).

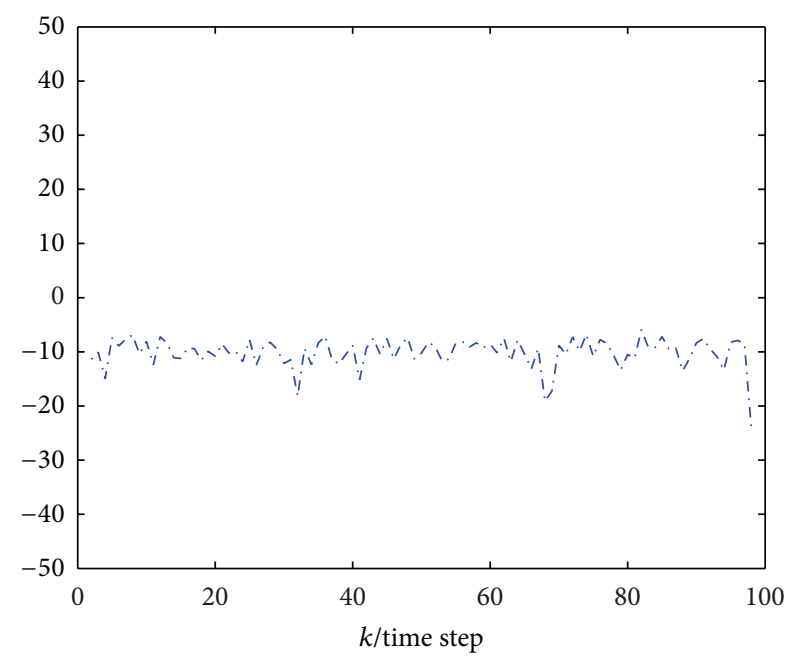

Figure 7: $\log (\mathrm{MSE1})$ (Case II).

\section{Conclusion}

The problem of the optimal Kalman filtering has been investigated for a class of linear discrete stochastic systems with multiplicative noises and random two-step sensor delays. Three Bernoulli distributed random variables with known conditional probabilities have been introduced to describe the phenomena of two-step sensor delays. Based on the innovation analysis approach and the recursive projection formula, for both the multiplicative noises and the random two-step sensor delays, a new optimal Kalman filtering has been proposed for the addressed linear stochastic system. Further research topics include the extension of the developed optimal filtering strategy to the prevalent eventtriggered case [35], more networked induced phenomena as in [36], and the random delays modeled by the Markov chain [37]. Moreover, it would be interesting and important to deal 


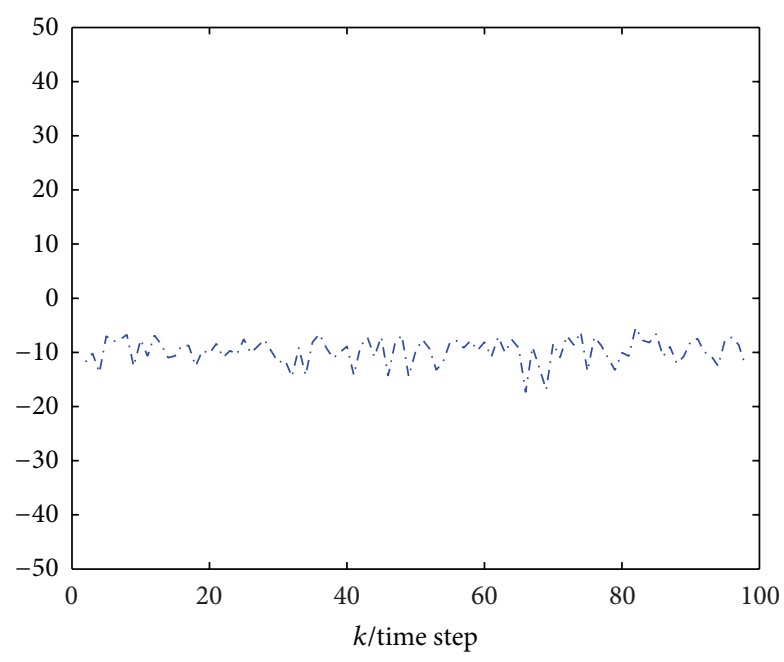

Figure 8: $\log ($ MSE2) (Case II).

with the stability analysis issue for the proposed filtering algorithm.

\section{Conflict of Interests}

The authors declare that there is no conflict of interests regarding the publication of this paper.

\section{Acknowledgments}

The authors would like to thank the associate editor and the anonymous reviewers for their detailed comments and valuable suggestions. This work was supported in part by the National Natural Science Foundation of China (NSFC) under Grants 11271103 and 11301118.

\section{References}

[1] M. Darouach and H. S. Ali, "Optimal unbiased functional filtering in the frequency domain," Systems Science \& Control Engineering, vol. 2, no. 1, pp. 308-315, 2014.

[2] J. Hu, Z. Wang, H. Gao, and L. K. Stergioulas, "Probabilityguaranteed $H_{\infty}$ finite-horizon filtering for a class of nonlinear time-varying systems with sensor saturations," Systems \& Control Letters, vol. 61, no. 4, pp. 477-484, 2012.

[3] H. R. Karimi, M. Zapateiro, and N. Luo, "A linear matrix inequality approach to robust fault detection filter design of linear systems with mixed time-varying delays and nonlinear perturbations," Journal of the Franklin Institute, vol. 347, no. 6 , pp. 957-973, 2010.

[4] H. Dong, Z. Wang, S. X. Ding, and H. Gao, "Finite-horizon estimation of randomly occurring faults for a class of nonlinear time-varying systems," Automatica, vol. 50, no. 12, pp. 31823189, 2014.

[5] M. Z. A. Bhotto and I. V. Bajic, "Constant modulus blind adaptive beamforming based on unscented Kalman filtering," IEEE Signal Processing Letters, vol. 22, no. 4, pp. 474-478, 2015.
[6] H. Rezaei, M. Reza, and M. H. Sedaaghi, "Improved robust finite-horizon Kalman filtering for uncertain networked timevarying systems," Information Sciences, vol. 293, pp. 263-274, 2015.

[7] R. E. Kalman, "A new approach to linear filtering and prediction problems," Journal of Fluids Engineering, vol. 82, no. 1, pp. 35-45, 1960.

[8] J. Ma and S. Sun, "Optimal linear estimators for systems with random sensor delays, multiple packet dropouts and uncertain observations," IEEE Transactions on Signal Processing, vol. 59, no. 11, pp. 5181-5192, 2011.

[9] H.-X. Chen and S.-L. Sun, "Optimal linear estimators for networked control systems with finite consecutive packet dropouts," Control Theory and Applications, vol. 29, no. 10, pp. 1317-1324, 2012.

[10] T. Utz, C. Fleck, J. Frauhammer, D. Seiler-Thull, and A. Kugi, "Extended Kalman filter and adaptive backstepping for mean temperature control of a three-way catalytic converter," International Journal of Robust and Nonlinear Control, vol. 24, no. 18, pp. 3437-3453, 2013.

[11] Z. Wang, X. Liu, Y. Liu, J. Liang, and V. Vinciotti, "An extended Kalman filtering approach to modeling nonlinear dynamic gene regulatory networks via short gene expression time series," IEEE/ACM Transactions on Computational Biology and Bioinformatics, vol. 6, no. 3, pp. 410-419, 2009.

[12] J. Hu, Z. Wang, H. Dong, and H. Gao, "Recent advances on recursive filtering and sliding mode design for networked nonlinear stochastic systems: a survey," Mathematical Problems in Engineering, vol. 2013, Article ID 646059, 12 pages, 2013.

[13] K. Q. Nguyen, T. H. Nguyen, and Q. P. Ha, "FPGA-based sensorless PMSM speed control using reduced-order extendedKalman filters," IEEE Transactions on Industrial Electronics, vol. 61, no. 12, pp. 6574-6582, 2014.

[14] H. Ahmada and T. Namerikawa, "Extended Kalman filter-based mobile robot localization with intermittent measurements," Systems Science \& Control Engineering, vol. 1, no. 1, pp. 113-126, 2013.

[15] A. Ghanbari and M. Moghanni-Bavil-Olyaei, "Adaptive fuzzy terminal sliding-mode control of MEMS z-axis gyroscopewith extended Kalman filter observer "' Systems Science and Control Engineering, vol. 2, no. 1, pp. 183-191, 2014.

[16] J. Hu, Z. Wang, Y. Niu, and L. K. Stergioulas, “ $H_{\infty}$ sliding mode observer design for a class of nonlinear discrete time-delay systems: a delay-fractioning approach," International Journal of Robust and Nonlinear Control, vol. 22, no. 16, pp. 1806-1826, 2012.

[17] Y. Halevi and A. Ray, "Integrated communication and control systems. Part 1-analysis," Transactions of the ASME-Journal of Dynamic Systems, Measurement and Control, vol. 110, no. 4, pp. 367-373, 1988.

[18] L. Wang, G. Wei, and H. Shu, "State estimation for complex networks with randomly occurring coupling delays," Neurocomputing, vol. 122, pp. 513-520, 2013.

[19] A. Ray and Y. Halevi, "Integrated communication and control systems: part II-design considerations," Journal of Dynamic Systems, Measurement and Control, vol. 110, no. 4, pp. 374-381, 1988.

[20] J. Liang, B. Shen, H. Dong, and J. Lam, "Robust distributed state estimation for sensor networks with multiple stochastic communication delays," International Journal of Systems Science, vol. 42, no. 9, pp. 1459-1471, 2011. 
[21] J. Nilsson, B. Bernhardsson, and B. Wittenmark, "Stochastic analysis and control of real-time systems with random time delays," Automatica, vol. 34, no. 1, pp. 57-64, 1998.

[22] Y. Luo, G. Wei, X. Ding, and Y. Liu, "Controller design for 2D stochastic nonlinear Roesser model: a probability-dependent gain-scheduling approach," Journal of the Franklin Institute. Engineering and Applied Mathematics, vol. 351, no. 11, pp. 51825203, 2014.

[23] J. Hu, D. Chen, and J. Du, "State estimation for a class of discrete nonlinear systems with randomly occurring uncertainties and distributed sensor delays," International Journal of General Systems, vol. 43, no. 3-4, pp. 387-401, 2014.

[24] J. Liang and J. Cao, "Global output convergence of recurrent neural networks with distributed delays," Nonlinear Analysis: Real World Applications, vol. 8, no. 1, pp. 187-197, 2007.

[25] J. Liang, Z. Wang, Y. Liu, and X. Liu, "State estimation for twodimensional complex networks with randomly occurring nonlinearities and randomly varying sensor delays," International Journal of Robust and Nonlinear Control, vol. 24, no. 1, pp. 1838, 2014.

[26] L. Wang, G. Wei, and W. Li, "Probability-dependent $H_{\infty}$ synchronization control for dynamical networks with randomly varying nonlinearities," Neurocomputing, vol. 133, pp. 369-376, 2014.

[27] S. L. Sun, "Optimal linear estimation for networked systems with one-step random delays and multiple packet dropouts," Acta Automatica Sinica, vol. 38, no. 3, pp. 349-356, 2012.

[28] S. Sun, L. Xie, W. Xiao, and N. Xiao, "Optimal filtering for systems with multiple packet dropouts," IEEE Transactions on Circuits and Systems II: Express Briefs, vol. 55, no. 7, pp. 695-699, 2008.

[29] J. Hu, Z. Wang, B. Shen, and H. Gao, "Gain-constrained recursive filtering with stochastic nonlinearities and probabilistic sensor delays," IEEE Transactions on Signal Processing, vol. 61, no. 5, pp. 1230-1238, 2013.

[30] D. Chen and L. Xu, "Optimal Kalman filtering for a class of state delay systems with randomly multiple sensor delays," Abstract and Applied Analysis, vol. 2014, Article ID 716716, 10 pages, 2014.

[31] A. Hermoso-Carazo and J. Linares-Pérez, "Unscented filtering algorithm using two-step randomly delayed observations in nonlinear systems," Applied Mathematical Modelling: Simulation and Computation for Engineering and Environmental Systems, vol. 33, no. 9, pp. 3705-3717, 2009.

[32] J. Feng, Z. Wang, and M. Zeng, "Optimal robust non-fragile Kalman-type recursive filtering with finite-step autocorrelated noises and multiple packet dropouts," Aerospace Science and Technology, vol. 15, no. 6, pp. 486-494, 2011.

[33] J. Hu, Z. Wang, B. Shen, and H. Gao, "Quantised recursive filtering for a class of nonlinear systems with multiplicative noises and missing measurements," International Journal of Control, vol. 86, no. 4, pp. 650-663, 2013.

[34] O. L. V. Costa and G. R. A. M. Benites, "Linear minimum mean square filter for discrete-time linear systems with Markov jumps and multiplicative noises," Automatica, vol. 47, no. 3, pp. 466476, 2011.

[35] S. Trimpe and R. D’Andrea, "Event-based state estimation with variance-based triggering," IEEE Transactions on Automatic Control, vol. 59, no. 12, pp. 3266-3281, 2014.

[36] Z. Wang, H. Dong, B. Shen, and H. Gao, "Finite-horizon $H_{\infty}$ filtering with missing measurements and quantization effects," IEEE Transactions on Automatic Control, vol. 58, no. 7, pp. 1707$1718,2013$.
[37] M. J. García-Ligero, A. Hermoso-Carazo, and J. Linares-Pérez, "Distributed fusion estimation in networked systems with uncertain observations and Markovian random delays," Signal Processing, vol. 106, pp. 114-122, 2015. 


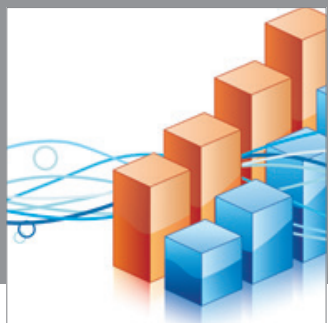

Advances in

Operations Research

mansans

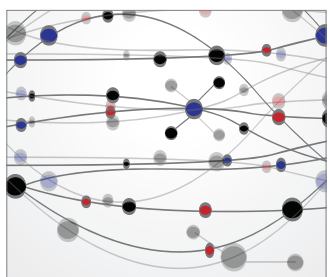

The Scientific World Journal
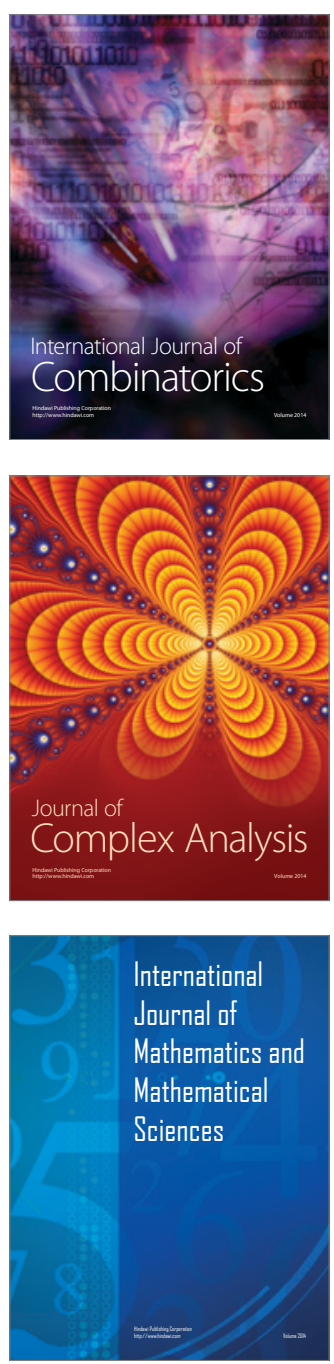
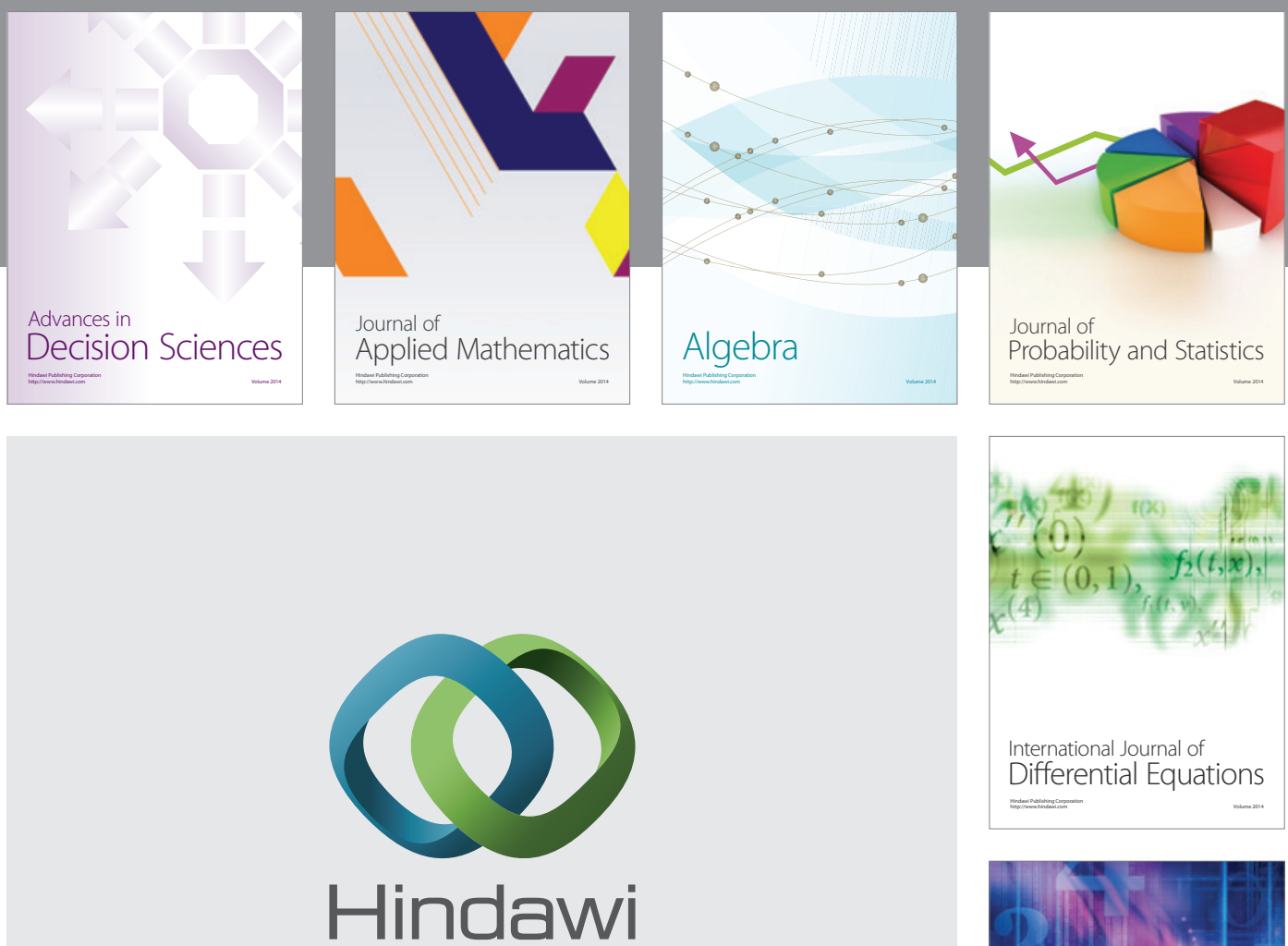

Submit your manuscripts at http://www.hindawi.com
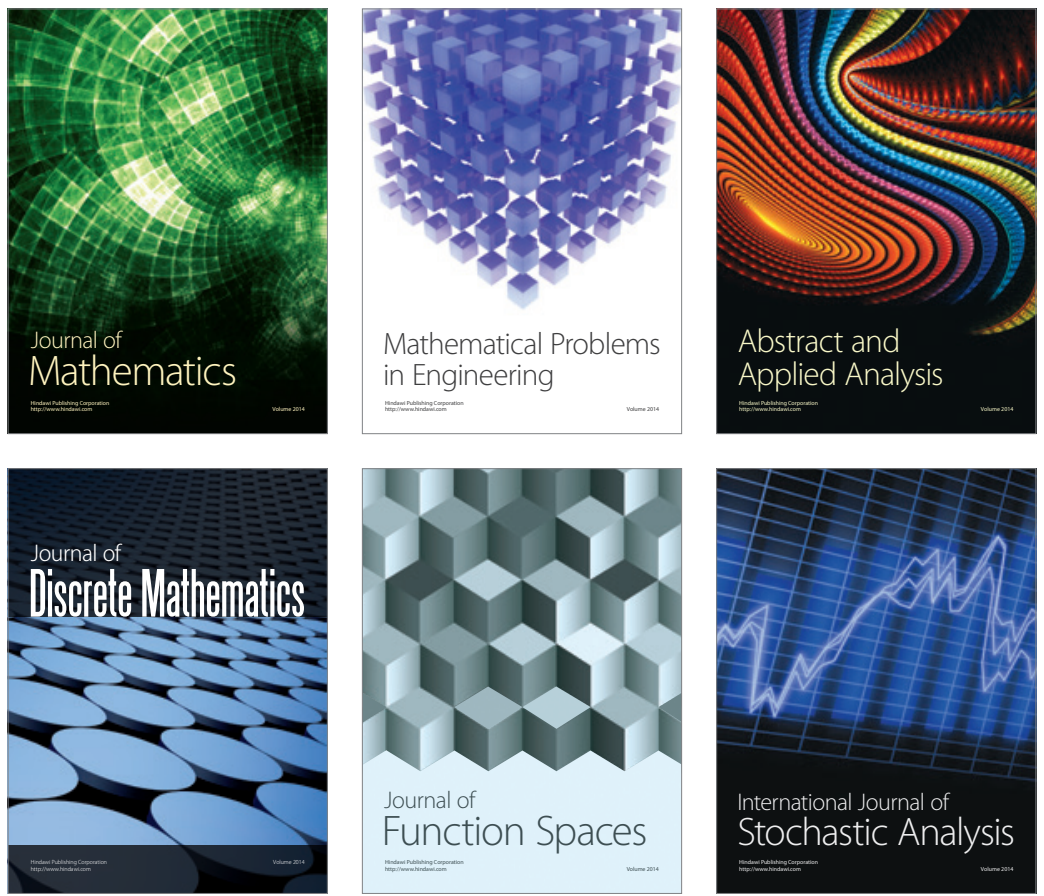

Journal of

Function Spaces

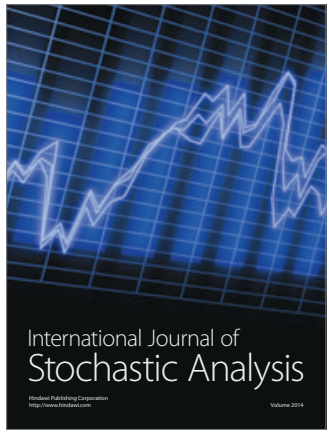

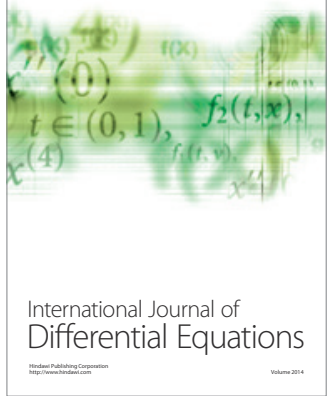
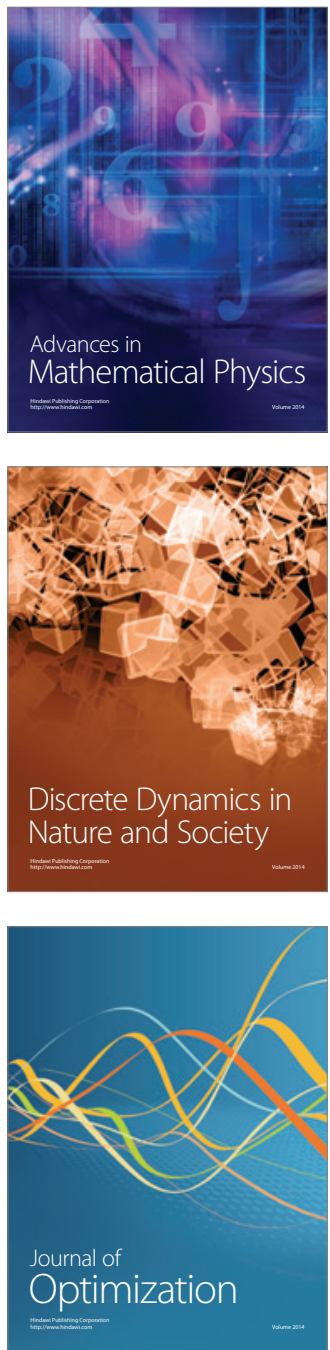